\title{
Acknowledgment of Reviewers
}

The Editors depend heavily on outside reviewers in forming opinions about papers submitted to JNeurosci and would like to formally thank the following individuals for their help during the past year.

Esther Aarts

Ishmail Abdus-Saboor

Ted Abel

Moshe Abeles

Aman Aberra

Wickliffe C. Abraham

Andrey Y. Abramov

Thomas W. Abrams

Brian D. Ackley

Laszlo Acsady

David Jean Acunzo

Chinnakkaruppan Adaikkan

Antoine Adamantidis

Patti Adank

Avishek Adhikari

Hita Adwanikar

Dritan Agalliu

Amit Agarwal

Ehud Ahissar

Merav Ahissar

Seppo Ahlfors

Susanne E. Ahmari

Zaghloul Ahmed

Jyrki Ahveninen

Mikko Airavaara

Thomas Akam

Aidas Alaburda

Andrea Alamia

F. Xavier Alario

Mohsen Alavash

Dinu Florin Albeanu

Anke Marit Albers

Urs Albrecht

Hakan Aldskogius

Zane Nathan Aldworth

David Murray Alexander

Simon Alford

Amber L. Alhadeff

Douglas Watt Allan

Andrew Mark Allen

Charles N. Allen

Micah Allen

Timothy A. Allen

Máté Aller

Brian Allman

Jorge Almeida

Jose Manuel Alonso

Eckart Altenmüller

Francisco J. Alvarez

Daniel Alvarez Fischer

Céline Amiez

Alon Amir

Konstantinos Ampatzis

Costas A. Anastassiou

Mark L. Andermann

Adam Keith Anderson

Aileen J. Anderson

Anne E. Anderson

Michael C. Anderson

Samira Anderson

Stewart A. Anderson

Anuska V. Andjelkovic

Rodrigo Andrade
Tim Andrews

Alessandra Angelucci

María Cecilia Angulo

Lucio Annunziato

Josef Anrather

Michelle Antoine

Stefano Anzellotti

Sho Aoki

Jason Aoto

Matthew Alexander

James Apps

Ken Arai

Toshiyuki Araki

Ottavio Arancio

Gordon W. Arbuthnott

Michael J. Arcaro

Benjamin Arenkiel

Juan Carlos Arevalo

William Armstrong

Amy F. T. Arnsten

Adam Robert Aron

Maithe Arruda-Carvalho

Jonathan Art

Shahar Arzy

Wael F. Asaad

James Ashe

Yoshinori Aso

Arun Asok

Gary Aston-Jones

Simone Astori

Deniz Atasoy

William Atchison

Huriye Atilgan

Lauren Atlas

Craig S. Atwood

Ryszard Auksztulewicz

Vanessa J. Auld

Guy Avraham

Rajeshwar Awatramani

Edward Awh

Vadim Axelrod

Scott Ayton

Peter W. Baas

Kavita Babu

Dominik Bach

Adam D. Bachstetter

Ryan K. Bachtell

Kristina C. Backer

Alexandra Badea

Tom Baden

David Badre

Wolfgang Baehr

Martha Bagnall

Mathias Bähr

Herwig Baier

Jaideep Singh Bains

Roland Bainton

Wyeth Bair

Chris Ian Baker

Daniel H. Baker

Stuart N. Baker

Priti Balchandani

Tracy L. Bale
Timothy S. Balmer

Joshua Balsters

Shernaz X. Bamji

Swati Banerjee

Debra A. Bangasser

Michael Mario Bannert

Jianxin Bao

Shaowen Bao

Jay Baraban

Segev Barak

Tallie Z. Baram

Helen Barbas

Deanna Barch

Annamaria Barczak

Yves-Alain William Barde

Barbara Bardoni

Sebastiano Bariselli

Edi Barkai

Tania Rinaldi Barkat

David James Barker

Gareth R. I. Barker

Jacqueline M. Barker

Linda A. Barlow

Carol A. Barnes

Alexander J. Barnett

Susan Carol Barnett

Colin J. Barnstable

Frank C. Barone

Ruth M. Barrientos

Luis Felipe Barros

Jeremy Barry

Alison L. Barth

Peter Bartho

Edward Lee Bartlett

Ramon Bartolo

Paolo Bartolomeo

Pablo Barttfeld

Benjamin M. Basile

Andrew Bass

Joshua P. Bassett

D. Michele Basso

Manuela Basso

Michele A. Basso

Brock Bastian

Hélène Bastuji

Emily Anne Bates

Helen S. Bateup

Brice Bathellier

Agnès Baude

Michel Baudry

Markus Bauer

Mark G. Baxter

K. Ulrich Bayer

Maxim Bazhenov

M. Flint Beal

Bruce P. Bean

Marco Beato

Christian Beaulieu

Heinz Beck

Catherina G. Becker

Jill B. Becker

Laren Becker

Thomas Becker
Michael J. Beckstead

Marina Bedny

Anton Ludwig Beer

Simon Beggs

Marlene Behrmann

Roozbeh Behroozmand

Kevin Beier

Jean-Claude Beique

Florian Beissner

Pascal Belin

Buddhika Bellana

Hugo J. Bellen

Jacob Bellmund

Riccardo Beltramo

Kevin J. Bender

Daniel Bendor

Tamir Ben-Hur

Tim A. Benke

James P. Bennett

Matthew Bennett

Larry I. Benowitz

Yehuda Ben-Shahar

Deanna L. Benson

Andrea Benucci

Helene Benveniste

Aya Ben-Yakov

Yulia Bereshpolova

Rune W. Berg

Joshua Berke

Sarah B. Berman

Olivia Bermingham-McDonogh

Christophe Bernard

Paolo Bernardi

Boris Bernhardt

Pierre-Michel Bernier

Benedikt Berninger

Wade H. Berrettini

Craig Berridge

Kent Berridge

David Berron

Jacob A. Berry

David M. Berson

Luciana Besedovsky

Christian Beste

Sven Bestmann

J. Nicholas Betley

Bernhard Bettler

Richard F. Betzel

Marianne Bezaire

Ilya Bezprozvanny

Paola Bezzi

Apoorva Bhandari

Manzoor A. Bhat

Anita Bhattacharyya

Roberta Bianco

Martha Elise Bickford

Kasia M. Bieszczad

Sandra Billinger

Lynne Bilston

Ulrike Bingel

Ferdinand Binkofski

Carmen Birchmeier

James A. Bisby 
Josef Bischofberger

Eric Bittman

He Biyu

Jennifer Kim Bizley

L. Ashley Blackshaw

Seth Blackshaw

Craig Blackstone

Mark Blagrove

Laura J. Blair

David T. Blake

Randy D. Blakely

Thomas A. Blanpied

Justin Blau

Julie Ann Blendy

Armin Blesch

Dawn M. Blitz

Mark S. Blumberg

Mathew Blurton-Jones

Davi D. Bock

Róbert Bódizs

Henning Boecker

Darren Boehning

Paul Bolam

Vadim Bolshakov

Mélanie Boly

Luis Bonet-Ponce

Luke W. Bonham

Patricia Bonnavion

Mathilde Bonnefond

Sam A. Booker

Tjeerd W. Boonstra

Erie Dell Boorman

David R. Borchelt

Angelique Bordey

Bart Gerard Borghuis

Alla Borisyuk

Cesar Borlongan

Aaron M. Bornstein

Gerard Borst

Daniele Borzelli

Conrado Bosman

Jennifer Marie Bossert

Roberto Bottini

Kristofer E. Bouchard

Sebastien Bouret

Jennifer Nicole Bourne

Charles W. Bourque

Anne-Laurence Boutillier

Derek Bowie

Thomas Bozza

Yuri Bozzi

Stefania Bracci

Senne Braem

Darrin H. Brager

David Brainard

Michael S. Brainard

Bob Bramson

Marco Brancaccio

Talia Brandman

Marko Brankatsch

Jan Willem Brascamp

Marcel Brass

Christian Bravo-Rivera

Timothy Bredy

Kellie Breen

Frank Bremmer

Heather C. Brenhouse

Jonathan Brennan
Robert Brenner

Assaf Breska

Vincent Breton-Provencher

Frederic Bretzner

Amy L. Brewster

Melinda Brindley

Jonathan P. Britt

Kendal Broadie

Christian Broberger

Christian Brodbeck

Michael Paul Broderick

Ethan S. Bromberg-Martin

Nils Brose

Craig E. Brown

Peter Brown

Ritchie Edward Brown

Steven A. Brown

Michael Browning

Thomas J. Brozoski

Michael Bruchas

Rose Bruffaerts

Norbert Bruggemann

Joshua C. Brumberg

Nicolas Brunel

Patrick Bruns

Bingni W. Brunton

Nickolay Brustovetsky

Macià Buades-Rotger

Dirk Bucher

Joshua W. Buckholtz

Paul Buckmaster

Thomas Budde

Annalisa Buffo

Tuan V. Bui

Daniel Bullock

Christopher Alan Buneo

Silvia A. Bunge

Nico Bunzeck

Andres Buonanno

Dean V. Buonomano

Denis Burdakov

Etienne Burdet

Tricia H. Burdo

Christian Richard Burgess

Robert W. Burgess

Sara N. Burke

Marie Burns

Brian Donald Burrell

Rami Burstein

Edward A. Burton

Niko A. Busch

Daniel Bush

Blake Edward Butler

Simon J. B. Butt

D. Allan Butterfield

Laurel Buxbaum

Winston D. Byblow

Margaret S. Bynoe

Hayriye Cagnan

Catherine M. Cahill

Denise Cai

Qian Cai

Christopher Kenneth Cain

Scott Ashley Cairney

Brian Edwin Cairns

Ronald L. Calabrese

Vittorio Calabrese

Paolo Calabresi
Nicole Calakos

Guy A. Caldwell

John Caldwell

Kim A. Caldwell

Federico Calegari

Matteo Caleo

Gwendolyn G. Calhoon

David J. Calkins

Bridget Laura Callaghan

Martina F. Callaghan

Frédéric Calon

Peter Deane Calvert

Heather A. Cameron

Roberto Caminiti

Wendy Marie Campana

Karen Lucia Campbell

Kenneth Campbell

Rebecca Campbell

Daniel Campbell-Meiklejohn

Peter A. Campochiaro

Carmen Castro Canavier

Laura Cancedda

Matteo Candidi

Marco Canepari

Jessica F. Cantlon

Ling Cao

Peng Cao

Marco Capogna

Matteo Carandini

Melissa L. Caras

W. Shawn Carbonell

Jessica A. Cardin

Jean-René Cardinaux

Albert Cardona

Astrid Cardona

Regina M. Carelli

Thomas J. Carew

Megan R. Carey

Johan Daniel Carlin

Thomas A. Carlson

David Carmel

S. Thomas Carmichael

Laurel H. Carney

Yarimar Carrasquillo

Manuel Carreiras

Dr. C. Carroll

Brett C. Carter

Bruce D. Carter

Gemma Carvill

Christian Casanova

Valerie Castellani

Anna Castelnovo

Pablo Enrique Castillo

Michael J. Caterina

Carmen Cavada

James Cavanagh

John W. Cave

Michel Cayouette

Dario Cazzoli

Pablo A. Celnik

Angela Cenci

Maria Fernanda Ceriani

Leonardo Cerliani

Maurice J. Chacron

Paul Chadderton

Matthew V. Chafee

Maria Chahrour

Maria Chait
Sumana Chakravarty

Savio Chan

Lawrence Judson Chandler

Anand Chandrasekhar

Catie Chang

Kae-Jiun Chang

Le Chang

Steve Wohn Chul Chang

Edwin R. Chapman

Caroline Juliette Charpentier

Elena Chartoff

Henry Chase

Robert S. Chavez

Charles Chavkin

Alain Chedotal

Bin Chen

Chinfei Chen

Hsiao-Jou Cortina Chen

Jeannie Chen

Lu Chen

Robert Chen

Xiaoke Chen

Alan G. Cheng

Alex Chesler

Mitchell Chesler

Vincent Chi Kwan Cheung

Douglas Cheyne

Ann-Shyn Chiang

Isaac Chiu

Kyung-Ok Cho

Sunghee Cho

Inyong Choi

Shen-Ju Chou

Rumana Chowdhury

Anastasia Christakou

Brian R. Christie

Jason M. Christie

Evangelos Christou

James Joseph Chrobak

Evangelia G. Chrysikou

Charleen Chu

Yogita Chudasama

ChiHye Chung

Dongil Chung

Man Kyo Chung

Moo Chung

Roberto Ciccocioppo

Radoslaw M. Cichy

Stéphane Ciocchi

Philippe Ciofi

Chiara Cirelli

Damon Clark

Alex Clarke

Joseph Classen

Roger L. Clem

David Clewett

Hollis T. Cline

Jean-François Cloutier

Thomas M. Coate

Luke Coddington

Ruben Coen-Cagli

Jeremiah Cohen

Jessica R. Cohen

Laurent Cohen

Ben Colagiuri

Roger J. Colbran

Michael W. Cole

Jonathan Ainsley Coles 
Olivier Collignon

Thérèse Collins

Christopher S. Colwell

Barry G. Condron

Barry W. Connors

Christos Constantinidis

Bruno Conti

Anis Contractor

Diego Contreras

Bevil R. Conway

Jennifer Cook

Mark James Cook

Sam Cooke

Mark Cookson

Stephen Coombes

Patrick Cooper

Thomas Cope

Mathieu Coppey

Richard Coppola

Laura H. Corbit

Gabriel Corfas

Brian D. Corneil

Pierre-Jean Corringer

Rosa Cossart

Rui Ponte Costa

Vincent Costa

Mauro Costa-Mattioli

Marcello Costantini

Susan Cotman

Julien Courchet

Michael Alan Cousin

Rita M. Cowell

Rosemary A. Cowell

Stephen L. Cowen

Brandon Cox

Roy Cox

Joseph T. Coyle

Ann-Marie Craig

Andre Mascioli Cravo

Meaghan Creed

Harold Cremer

Maite Crespo-Garcia

Frédéric Crevecoeur

Fulton T. Crews

Sylvain Crochet

Stephen J. Crocker

Michael A. Crognale

Elizabeth Carol Cropper

Scott J. Cruikshank

Ana V. Cruz

Jozsef Csicsvari

Jody C. Culham

Bruce G. Cumming

Paul Cumming

Theodore R. Cummins

Sebastian Curti

Clayton E. Curtis

Carina Curto

Rhodri Cusack

Istvan Czigler

Alain Dabdoub

Christopher J. Dakin

Turgay Dalkara

Matthew B. Dalva

Numa Dancause

Claudia Danielmeier

Valerie Darcey

Corinna J. Darian-Smith
Warren Darling

Aniruddha Das

Jeremy Dasen

Christoph Daube

Ana M. Daugherty

Andrea d'Avella

Samuel David

Beverly L. Davidson

Brian M. Davis

Jeffrey Parra Davis

Ronald L. Davis

Simon W. Davis

Nathaniel D. Daw

Ted M. Dawson

Jeremy J. Day

Mitchell L. Day

Eran Dayan

Ivan E. de Araujo

Lindsay De Biase

Will de Cothi

Jan Willem de Gee

Tom Alexander de Graaf

Marzia De Lucia

Natalia De Marco Garcia

Benedetto De Martino

Joriene C. De Nooij

Lucas de Oliveira Alvares

Dominique J. F. De Quervain

Didier De Saint Jan

Étienne de Villers-Sidani

Lycia Dieneke de Voogd

Joris de Wit

Chris I. De Zeeuw

Douglas C. Dean

Michael R. Deans

Dominique Debanne

Stefan Debener

Jay Debnath

Tessa Dekker

Daniel del Toro

Kristen Delevich

Patrick Delmas

Gianpaolo Demarchi

Athena Demertzi

Ece Demir-lira

Laura A. DeNardo

Benjamin Deneen

Evan S. Deneris

Rachel N. Denison

Fatma Deniz

Bryan Denny

Christine A. Denny

Erik W. Dent

Andre Der-Avakian

Dori Derdikman

Niraj S. Desai

Kobe Desender

Mohanish Deshmukh

Tara M. DeSilva

Anne Desmazières

Aline Desmedt

Mark D'Esposito

Theresa M. Desrochers

Lakshmi A. Devi

Steven H. DeVries

Ajay Dhaka

Graziella Di Cristo

Rossella Di Giaimo
Giuseppe Di Giovanni

Vincenzo Di Lazzaro

Giovanni M. Di Liberto

Patricia M. Di Lorenzo

Giuseppe di Pellegrino

Andreea Oliviana Diaconescu

Begoña Díaz

Sulayman D. Dib-Hajj

Frederic Dick

Dion K. Dickman

Jörn Diedrichsen

Cristina V. Dieni

Herman Dierick

David M. Dietz

Martin J. Dietz

Derk-Jan Dijk

Nadine Dijkstra

Ralph DiLeone

Olaf Dimigen

Jun Ding

Long Ding

Nai Ding

Xiao-Qi Ding

Heinrich Dircksen

Anita Disney

John Disterhoft

Jochen Ditterich

Jeremy S. Dittman

Michael Tri Hoang Do

Lauren Dobbs

Katharina Dobs

Paul Dodson

Chris Q. Doe

Christian Doeller

Keith B. Doelling

Florin Dolcos

Patrick J. Dolph

Annette C. Dolphin

Manus J. Donahue

Bruce Dore

Alan D. Dorval

Nico Urs Felix Dosenbach

Kimberly J. Dougherty

Patrick M. Dougherty

Paul Downing

Andreas Draguhn

Alex Dranovsky

Jens P. Dreier

Uwe Drescher

Michael R. Drew

Trevor Drew

Cheryl F. Dreyfus

Jan Drugowitsch

Janet M. Dubinsky

Jessica Dubois

Sarah DuBrow

Edward Dudek

Serena M. Dudek

Laura Dugué

Chris G. Dulla

Didier Dulon

Ronald S. Duman

Serge O. Dumoulin

John Duncan

Volker Dürr

Isabelle Dusart

Mathias Dutschmann

Éléonore Duvelle
Paul Edmund Dux

Jacques Duysens

Michael Dyer

Volodymyr Dzhala

Timothy J. Ebner

Richard A. E. Edden

Jean-Marc Edeline

V. Reggie Edgerton

Grace Edwards

Robert H. Edwards

Seth Egger

Erika D. Eggers

Tobias Egner

Martin Eimer

Wolfgang Einhäuser

Amelia J. Eisch

Marc Ekker

Fanny M. Elahi

Rodger J. Elble

Mark A. G. Eldridge

Felice Elefant

Ype Elgersma

Ana Belén Elgoyhen

Carol F. Elias

Stella Elkabes

Kate L. Ellacott

Keith S. Elmslie

Ben Emery

Patrick Emery

Uzay Emir

David Engblom

Stephen A. Engel

Tatiana Engel

Britta Engelhardt

Jan Engelmann

Michel Engeln

Rune Enger

Florian Engert

Peter Enticott

Bastian Epp

Jonathan Epstein

Julia Erb

Cagla Eroglu

Reha S. Erzurumlu

Carles Escera

Oxana Eschenko

Monique Esclapez

Rodrigo A. España

Steven Estus

Iryna M. Ethell

Chris Evans

Jennifer A. Evans

Rebekah Evans

Stefan Everling

Laura A. Ewell

Debra Ann Fadool

Gordon L. Fain

Scott L. Fairhall

Arnaud Y. Falchier

Terje Falck-Ytter

Sean James Fallon

Stephen Fancy

Frank M. Faraci

Alessandro Farnè

Mark Farrant

Lorna Farrelly

Patrizia Fattori

Victor Faundez 
James P. Fawcett

Mel B. Feany

Howard J. Federoff

Kerstin Feistel

Donna M. Fekete

Gordon Benedikt Feld

David A. Feldheim

Dirk Feldmeyer

Markus Fendt

Gangyi Feng

Jian Feng

Susan Marie Ferguson

Laura Marie Justine Fernandez

Paul Fernyhough

Stefano Ferraina

Joseph R. Fetcho

Christopher Fetsch

Todd A. Fiacco

André Fiala

Ian C. Fiebelkorn

Greg D. Field

Howard L. Fields

Maria E. Figueiredo-Pereira

Ione Fine

Peter Stephen Barss Finnie

Bente Finsen

Vincenzo G. Fiore

Bonnie Lynne Firestein

Adrian Georg Fischer

Jozsef Fiser

Gordon Fishell

Robert Fisher

Una Fitzgerald

Shelly B. Flagel

Roland W. Fleming

Stephen M. Fleming

Paul C. Fletcher

Steven Fliesler

Adeen Flinker

Stan B. Floresco

Eckart Foerster

Stuart Fogel

Csaba Földy

John Foley

Pamela L. Follett

Andrew Forge

Ian D. Forsythe

Eric S. Fortune

Jennifer Foss-Feig

Joshua J. Foster

Haleh Fotowat

Alyson E. Fournier

Michael A. Fox

Tom Francart

Michael Francis

Polytimi Frangou

C. Andrew Frank

Loren M. Frank

Paul Franken

Tom P. Franken

David W. Franklin

Robin J. M. Franklin

Kevin M. Franks

Kurt M. Fraser

Stefan Frässle

Charles J. Frazier

Michael A. Freed

John H. Freeman
Erez Freud

Christopher Fricke

Desdemona Fricker

Wilma J. Friedman

Rainer W. Friedrich

Alain Frigon

Robert Crooks Froemke

Gregory I. Frolenkov

Sascha Frühholz

Meng-Meng Fu

Julie L. Fudge

Lluís Fuentemilla

Peter Gerard Fuerst

Sabine Fuhrmann

Fumino Fujiyama

Izumi Fukunaga

Miquel A. Fullana

Patrick M. Fuller

Andrew Furley

Tomoyuki Furuyashiki

Babette Fuss

Anthony H. Futerman

Christopher V. Gabel

John Gabrieli

Brendan Gaesser

Steffen Gais

Aristea S. Galanopoulou

Joseph M. Galea

Michela Gallagher

Jack L. Gallant

Juan Álvaro Gallego

Claudio Galletti

Thierry Galli

Jason Gallivan

Adriana Galvan

Frederic Gambino

Karen L. Gamble

Paul Gamlin

Sunil P. Gandhi

Karunesh Ganguly

Julia Ganz

Richard Gao

Wei Gao

Xiao-Bing Gao

Alfredo J. Garcia

L. Rene Garcia

Paul Garcia

Adolfo García

Jaime García-Añoveros

Matthew P. H. Gardner

Esther Polinsky Gardner

Douglas D. Garrett

Paul Garrity

John Garthwaite

Mona M. Garvert

Sergio Gascón

Patricia Gaspar

Laurent Gautron

Shaoyu Ge

Woo-Ping Ge

Po-Wu Gean

Tamar Gefen

Maria Neimark Geffen

Howard E. Gendelman

Joy J. Geng

John Carib Gensel

Lisa Genzel

Bertram Gerber
Samuel J. Gershman

Michael D. Gershon

Damien Gervasoni

Jason Gestwicki

Ulrik Gether

Alireza Gharabaghi

Simona Ghetti

Veronica Ghiglieri

Geoffrey M. Ghose

Barry Giesbrecht

Karl Peter Giese

Roman J. Giger

Marieke R. Gilmartin

Nicholas W. Gilpin

Ed Giniger

Stephen D. Ginsberg

Alan R. Gintzler

Bruno Giordano

Gabrielle Girardeau

Simon Francis Giszter

Aaron D. Gitler

Maurizio Giustetto

Johannes Gjerstad

Julijana Gjorgjieva

Stefan Glasauer

Jonathan David Glass

Laurie S. Glezer

Elisabeth Glowatzki

Yukiko Goda

Laurent Goffart

Andre M. Goffinet

Nadine Gogolla

Michael S. Gold

Ethan Michael Goldberg

Jesse H. Goldberg

Joshua A. Goldberg

Michael E. Goldberg

Sam Adler Golden

Ariel Yeuhda Goldstein

Franz Goller

Julie D. Golomb

Peyman Golshani

Flávia Carvalho Alcantara Gomes

Jesse Gomez

Timothy M. Gomez

María Eugenia Gomez-Casati

Marta Gomez-Galan

Hiroaki Gomi

J. Tiago Goncalves

Travis David Goode

Lisa V. Goodrich

Joshua A. Gordon

Robbe L. T. Goris

Jan A. Gorter

Simon Gosgnach

Yukiko Gotoh

Jacqueline Gottlieb

Elizabeth Gould

Thomas W. Gould

Todd D. Gould

Gunnar K. Gouras

Olivier Goureau

Boris Gourévitch

Juan D. Goutman

Alexander Gow

David Gow

Laetitia Grabot

Elena Gracheva
Viviana Gradinaru

Cheryl L. Grady

Scott T. Grafton

Joanes Grandjean

Jorg Grandl

David R. Grattan

Caterina Gratton

Ari J. Green

David W. Green

Steven Greenberg

Joshua D. Greene

Ian Greenhouse

Mark W. Greenlee

Charles A. Greer

Paul Greer

Ursula Greferath

Christian Grefkes

Georgia Gregoriou

Tineke Grent-'t-Jong

Pierre Gressens

Jan Grewe

Timothy David Griffiths

Warren M. Grill

Valery Grinevich

Iris I. A. Groen

Stephanie Mary Groman

Tijl Grootswagers

Benedikt Grothe

Kevin L. Grove

Andrew K Groves

Matthew S. Grubb

Aaron J. Gruber

Matthias J. Gruber

Rebecca Grzadzinski

Jianguo $\mathrm{Gu}$

Yong Gu

William Guido

Marc Guitart-Masip

Rasa Gulbinaite

Allan T. Gulledge

Christopher Gundlach

Jenny Gunnersen

J. Swaroop Guntupalli

Stephanie L. Gupton

Sarah Guthrie

Michael J. Gutnick

Alexander Gutschalk

Patrice Guyenet

Laura Gwilliams

Julie S. Haas

Troy A. Hackett

Daniel Hackman

Ziad M. Hafed

Steffen R. Hage

Stephen Haggarty

Pejmun Haghighi

Peter Hagoort

Bilal Haider

Adrian Mark Haith

Norbert Hajos

Michael M. Halassa

Tim Gerard Hales

Lindsay R. Halladay

Stefan Hallermannn

Geir Halnes

Edith Hamel

Arif A. Hamid

Antonia F. de C. Hamilton 
Liberty S. Hamilton

Peter J. Hamilton

Marc Hammarlund

Jin-Hee Han

Sung Han

Jonathan Gordon Hanley

Colleen A. Hanlon

Anthony John Hannan

Christian Hansel

Max F. K. Happel

Giles E. Hardingham

Carolyn W. Harley

Hala Harony-Nicolas

Alison Harris

Anthony Michael Harris

Kristen M. Harris

Peter Harrison

Gaylia Jean Harry

Catherine Hartley

Ben Mark Harvey

Monika Harvey

Andrea Hasenstaub

Michael Harvey Hastings

Nicholas George Hatsopoulos

Mary Elizabeth Hatten

Olaf Hauk

Lars Hausfeld

Anne Hauswald

Michael Hawken

Michael John Hawrylycz

Yasunori Hayashi

Phil Haydon

Seth Alanson Hays

Sheng $\mathrm{He}$

Zhigang He

Drew B. Headley

Luke James Hearne

Detlef H. Heck

Ellie S. Heckscher

Tobias Heed

Ruth Heidelberger

Cobi J. Heijnen

Stefan Heim

Grit Hein

Stephen J. Heinen

Randolph Frederik Helfrich

Moritz Helias

Aaron Shain Heller

Elizabeth Heller

Stefan Heller

Fritjof Helmchen

Michael Hendricks

Mark Henkemeyer

Linda Henriksson

Denise Henriques

Sophie K. Herbst

Melissa Herman

Jochen Herms

Eloisa Herrera

Björn Herrmann

Maria Del Carmen Herrojo Ruiz

Jeremy Herskowitz

Ronna Hertzano

Nora A. Herweg

David J. Herzfeld

Erik D. Herzog

Herbert Heuer

Robert F. Hevner
Shin-ichi Higashijima

Okihide Hikosaka

David L. Hill

Argye E. Hillis

Kristin L. Hillman

José Antonio Hinojosa

Simon Hippenmeyer

Yukinori Hirano

Judith A. Hirsch

James Hislop

Mark H. Histed

Peter F. Hitchcock

Thomas Scott Hnasko

Oliver Hobert

Patrick Hof

Jeremy Hogeveen

Avram J. Holmes

Todd C. Holmes

Jeffrey R. Holt

Matthew G. Holt

Anthony Holtmaat

David M. Holtzman

Erika Holzbaur

Jonathan Dean Hommel

Weizhe Hong

Sakiko Honjoh

Mark A. Hoon

Scott L. Hooper

Sarah C. Hopp

Gregory D. Horwitz

Howard A. Howard

Fleur Margaret Howells

Jason Howitt

Kari R. Hoyt

Liang-Tien Hsieh

Po-Jang Hsieh

Ming Hsu

Hailan $\mathrm{Hu}$

Chengcheng Huang

Eric J. Huang

Hao Huang

Helen J. Huang

Zirui Huang

Timothy Hubbard

Armin Huber

Daniel Huber

Andrew Hudson

Richard Lewis Huganir

Brent Hughes

Ethan G. Hughes

Frederick Hughson

John Huguenard

Alexander C. Huk

Court Hull

Hans R. Hultborn

Kathryn Humphreys

Laurence Hunt

Robert F. Hunt

James B. Hurley

Masud Husain

Cendri A. Hutcherson

Elizabeth Hutchinson

J. Benjamin Hutchinson

William Duncan Hutchison

Lara S. Hwa

James Michael Hyman

Steven E. Hyman

Costantino Iadecola
Giandomenico Iannetti

Guilhem Ibos

Ronaldo M. Ichiyama

Kei M. Igarashi

Kinga Iglói

Yuichi Iino

Satoshi Ikemoto

Tsuneya Ikezu

Peter Illes

Robin A. A. Ince

Artur Indzhykulian

Cory S. Inman

Nathan Insel

Karl John Iremonger

Muireann Irish

Tadashi Isa

Leyla Isik

Philippe Isope

Fadi A. Issa

Rutsuko Ito

Sirawaj Itthipuripat

Yuri P. Ivanenko

Jason J. Ivanusic

Alicia Izquierdo

Keise Izuma

Iiro P. Jääskeläinen

Anthony I. Jack

Skyler L. Jackman

Alexander C. Jackson

Meyer B. Jackson

Rebecca L. Jackson

Pierre-Yves Jacob

Heidi IL Jacobs

Joanna Jacobus

Shantanu P. Jadhav

Uday K. Jagadisan

Daniel J. Jagger

Bill Jagust

Marjan Jahanshahi

Varsha Jain

Sumitash Jana

Petr Janata

Dirk Jancke

Lutz Jancke

Elzbieta Jankowska

Joanna L. Jankowsky

Peter Janssen

Kyle Jasmin

Aaron M. Jasnow

Alexander Jaworski

Jacek Jaworski

Elizabeth Jefferies

Angela L. Jefferson

Paul M. Jenkins

Frances E. Jensen

Ole Jensen

James David Jentsch

Karim Jerbi

Thomas Clayton Jhou

Koji Jimura

Peng Jin

Gerhard Jocham

Daphna Joel

Joshua Johansen

Carolyn M. Johnson

Colin P. Johnson

Gail V. W. Johnson

Stephen M. Johnson
Wilsaan Mychal Joiner

Elizabeth Ann Jonas

Jacques Jonas

Peter Jonas

Matthew W. Jones

Theresa A. Jones

Thomas A. Jongens

James D. Jontes

Sven-Eric Jordt

Joshua B. Julian

David Julius

Rex Jung

Sandra Jurado

Vijay Mohan K. Namboodiri

Edor Kabashi

Leszek Kaczmarek

Pascal Simon Kaeser

Daniel Kaiser

John F. Kalaska

Zsuzsa Kaldy

Radha Kalluri

Kristjan Kalm

Maarten Kamermans

Karl Kandler

Pyungwon Kang

Patrick O. Kanold

Nancy Kanwisher

Aimee W. Kao

Mimi Kao

David M. Kaplan

Dimitrios Kapogiannis

Jaideep Kapur

Tim Karl

Haruo Kasai

Thomas Kash

Peter M. Kaskan

Sergey Kasparov

István Katona

David M. Katz

Donald B. Katz

Greta Kaufeld

Daniela Kaufer

Matthew Tyler Kaufman

Ege T. Kavalali

Kendrick N. Kay

Leslie M. Kay

Rakez Kayed

Andrew Kayser

Janet R. Keast

Kristen A. Keefe

Vladimir J. Kefalov

Andreas Keil

Julian Keil

Anne Keitel

Christian Keitel

Krystyna Keleman

Georgios A. Keliris

Alexander Kell

Christoph Kellendonk

Georg Keller

Matthew W. Kelley

Simon P. Kelly

Justin W. Kenney

Daniel Kerschensteiner

Jane Keung

Zayd M. Khaliq

Mazen A. Kheirbek

Jibran Younis Khokhar 
Arkady Khoutorsky

Ole Kiehn

Amy E. Kiernan

Rogier A. Kievit

Konstantina Kilteni

Clinton Kilts

Eunjoon Kim

Haesun A. Kim

Jun Hee Kim

Woo-Yang Kim

Koutarou D. Kimura

Katie Kindt

Mark S. Kindy

Andrew King

Sue C. Kinnamon

Lynne Kiorpes

Jonathan Kipnis

Elizabeth Kirby

Lynn G. Kirby

Alfredo Kirkwood

C. Brock Kirwan

Kirill Kiselyov

Johanna Kissler

Kazuo Kitamura

Anastasia Kiyonaga

Christian Klaembt

Avihu Klar

Andreas Klaus

Christine Klein

David Kleinfeld

Wolfgang Klimesch

Achim Klug

Floris Klumpers

Igor Klyubin

Tomas Knapen

David C. Knight

Robert Knight

Zachary Knight

Marlies Knipper

Thomas Knopfel

Thomas R. Knösche

Brian Knutson

Chien-Ping Ko

Han Seok Ko

Patrick Michael Kochanek

Peter Kochunov

Michael Koenigs

Paulo Kofuji

Kyunghee Koh

Stefan Köhler

Adam Kohn

Peter Kok

Erzsebet Kokovay

James Kolasinski

Anthony Koleske

Alex L. Kolodkin

Genevieve Konopka

Wouter Kool

Nancy Kopell

Christine Köppl

Christoph Korn

Martin Korte

Gyula Kovács

Yevgenia Kozorovitskiy

Brian C. Kraemer

Dimitri Krainc

John W. Krakauer

Andrej Kral
Helmut Kramer

Ulrike M. Krämer

David Evan Krantz

Michael J. Krashes

Alexander Kraskov

Eric Gerald Krause

Richard Krauzlis

Alexxai V. Kravitz

Gabriel Kreiman

Jens Kreitewolf

Bart Krekelberg

William S. Kremen

Esther Irene Krook-Magnuson

Leah Krubitzer

Kristine Krug

Hiroshi Kuba

Lubica Kubikova

James Kenneth Kubilus

Thomas Kuenzel

Brice Alan Kuhl

Arvind Kumar

Hrishikesh Kumar

Sukhbinder Kumar

Kazuhiko Kume

Chay T. Kuo

Min-Fang Kuo

David A. Kupferschmidt

Anna Kuppuswamy

Deborah Kurrasch

Peri Tamar Kurshan

Isaac Kurtzer

Christopher Kushmerick

Steven A. Kushner

Pawel Kusmierek

Jason J. Kutch

Janine L. Kwapis

Samuel Kwon

Anthony F. Lacagnina

Francesco Lacquaniti

Rosa Lafer-Sousa

Diane Carol Lagace

Leon Lagnado

Peter Lakatos

Edmund Lalor

Ryan T. LaLumiere

Anthony Samuel LaMantia

Nathalie Lamarche-Vane

Claus Lamm

Ilan Lampl

Raphael Lamprecht

Sofia M. Landi

Eric C. Landsness

Andrew P. Lane

Thomas E. Lane

Richard A. Lang

Angela Jane Langdon

Joachim Lange

Peter Langfelder

Dianne Langford

Markus Lappe

Michael Lardelli

Edward W. Large

Francesco Latini

K. Matthew Lattal

Helmut Laufs

Cornelia Laule

Jean Laurens

Gilles Laurent
Alexandra Lautarescu

Antonieta Lavin

Steven Robert LaViolette

Matthew J. LaVoie

Alpar Lazar

Orly Lazarov

Michael Lazarus

Ricardo Maurício Leao

Maël Lebreton

Magalie Lecourtois

Amy Lee

Andy C. H. Lee

Gloria Lee

Hyoung-gon Lee

Inah Lee

Soohyun Lee

Suk-Ho Lee

Taraz G. Lee

Yong-Seok Lee

Haemy Lee Masson

Julie L. Lefebvre

Michael Neal Lehman

Brendan Lehnert

Maria K. Lehtinen

Cynthia A. Lemere

Christian H. Lemon

Julia Cristine Lemos

Pierre-Pascal Lenck-Santini

Julia A. Leonard

Matthew K. Leonard

Jason P. Lerch

Holger Lerche

Mark D. Lescroart

Nicholas A. Lesica

Frances M. Leslie

Marcin Leszczynski

Paul C. Letourneau

Benedetta Leuner

Inge Leunissen

Daniel Leventhal

Martin Lévesque

Steven W. Levison

Edwin S. Levitan

Dino J. Levy

Penelope Lewis

R. S. Lewis

Jarrod A. Lewis-Peacock

Chris $\mathrm{Li}$

Jian Li

Ka Wan Li

Ling $\mathrm{Li}$

Nuo Li

Peng Li

Su Li

Xueting Li

Yang Li

Yuqing Li

Zhaoyu Li

M. Charles Liberman

Shane Liddelow

Andrew Lieberman

Cheng-Chang Lien

Romain Ligneul

Kyle P. Lillis

Daniel A. Lim

Janghoo Lim

Sung-Joo Lim

Huai-Ti Lin
Shih-Chieh Lin

Wensheng Lin

Jean-Marc Lina

David J. Linden

Jennifer F. Linden

Jon M. Lindstrom

Christopher D. Link

Klaus Linkenkaer-Hansen

Sara Linker

Michael Lippert

Diane Lipscombe

Ashok Litwin-Kumar

Robert C. Liu

Yunzhe Liu

Frederick J. Livesey

Britney Nola Lizama

Daniel A. Llano

María Llorens-Martín

Emyr Lloyd-Evans

Eng H. Lo

Patricia L. Lockwood

Daniel James Lodge

Terry Lohrenz

Michael Alan Long

Matthew R. Longo

Christophe Lopez

Enrique A. Lopez-Poveda

Jeffrey Lopez-Rojas

Hernan Lopez-Schier

Magor László Lörincz

Atilla Losonczy

Shahrdad Lotfipour

Joseph J. LoTurco

Malcolm James Low

Matthew X. Lowe

Pedro R. Lowenstein

Diego Lozano-Soldevilla

Hsin-Wei Lu

Q. Richard Lu

Xiao-Hong Lu

Joachim Heinz Rüdiger Lübke

Elizabeth K. Lucas

Steven J. Luck

Peter D. Lukasiewicz

Liqun Luo

Minmin Luo

Carl R. Lupica

Pierre-Hervé Luppi

Giuseppe Luppino

Gyorgy Lur

Christian Lüscher

Caroline Lustenberger

Patrick D. Lyden

David R. Lynch

David C. Lyon

David Lyons

Huan Ma

Le Ma

Minghong Ma

Tao Ma

Anne Maass

Nanna MacAulay

Gianmaria Maccaferri

Sean P. MacEvoy

M. Bruce MacIver

Kenneth Mackie

Wendy B. Macklin

Sandra Maday 
Daniel V. Madison

David Magnuson

Neil Stephen Magoski

Atul Maheshwari

Stephen Vincent Mahler

Alexander Maier

Joost X. Maier

Richard B. Mailman

Ania K. Majewska

Eugene Major

Carina Mallard

Nicolas Mallet

Manuel S. Malmierca

Francesca Maltecca

Manuel Mameli

Paul B. Manis

Jeremy Manning

Itzhak Mano

Dara S. Manoach

Patrick W. Mantyh

Yingwei Mao

Uri Maoz

Miguel Maravall

Eve Marder

Stephen Maren

Daniel Margoliash

Seth Shatkin Margolis

Eric Maris

Ulrika Marklund

Michelle Marneweck

Asif Maroof

Sean Marrett

Rogier Mars

Gary Marsat

Abigail Marsh

David Marshak

Lisa Marshall

Tom Rhys Marshall

Kirill A. Martemyanov

Alex Martin

Andrea E. Martin

Lee J. Martin

Loren Martin

Paul R. Martin

Marco Martina

Julio C. Martinez-Trujillo

Alain Marty

Michael Marxen

Mark A. Masino

Sotiris C. Masmanidis

Stephen C. Massey

Marcello Massimini

Mackenzie Mathis

Brian N. Mathur

Masanori Matsuzaki

Frederick R. Maxfield

Anton Maximov

Paul J. May

Helen Mayberg

Andrew Richard Mayes

Robin M. McAllen

David McAlpine

Chris J. McBain

Peter J. McCaffery

Margaret M. McCarthy

Jack McClellan

Timothy S. McClintock

Samuel McClure
Elizabeth A. McCullagh

Michael A. McDannald

Elizabeth A. McDevitt

Neil McDonald

Samuel David McDougle

Zoe A. McElligott

Braden C. McFarland

Sarah McFarlane

John P. McGann

Jill A. McGaughy

Aaron W. McGee

Matthew McGinley

Jacqueline Frances McGinty

Matt McHenry

Thomas J. McHugh

Anthony R. McIntosh

Theresa H. McKim

Rebecca Anne McKinney

Fiona McNab

Bruce L. McNaughton

Jared Medina

Paolo Medini

Sara Mednick

Julian P. Meeks

Lin Mei

Dies Meijer

Susanne Meis

Bartlett W. Mel

Carmen V. Melendez-Vasquez

Miriam Melis

Lucia Melloni

Caroline Menard

Juan Mena-Segovia

Lorne M. Mendell

Steven J. Mennerick

Vinod Menon

Karen Menuz

Manuel R. Mercier

Nicola B. Mercuri

M. Alex Meredith

Diane E. Merry

Charles Kenneth Meshul

Olimpia Meucci

Lars Meyer

Florent Meyniel

Michael Michaelides

Christoph M. Michel

John C. Middlebrooks

Gero Miesenböck

Michele Migliore

Mark J. Millan

Kathleen Joyce Millen

Dana Miller

Earl K. Miller

Karla L. Miller

Luke E. Miller

Mark W. Miller

Paul Miller

Richard J. Miller

Rebecca Elizabeth Millman

Gabriel Mindlin

Svjetlana Miocinovic

Veronica Miron

Zaman Mirzadeh

Markus Missler

Anna S. Mitchell

Daniel James Mitchell

Jude F. Mitchell
Makoto Mizunami

Dean Mobbs

Michelle Moerel

Sounak Mohanta

Marta Aragão Pacheco Moita

Sophie Molholm

Zoltan Molnar

Anna Molofsky

Valerie Mongrain

Kelly R. Monk

Philippe P. Monnier

Ilya E. Monosov

Read Montague

Daniela Montaldi

Lisa M. Monteggia

Martin M. Monti

Sally Moody

Richard Mooney

Brian C. J. Moore

Michele Morari

Robert Morecraft

Herman Moreno

Rubén Moreno-Bote

Gerardo Andres Morfini

Michael M. Morgan

Andrew T. Morgan

Josh M. Morganti

Shigeki Moriguchi

Hirofumi Morishita

Orson L. Moritz

John Morrey

Laurel Morris

Roger James Morris

Alessandro Moscateli

Matthew W. Mosconi

Morris Moscovitch

Ryan Mruczek

Liad Mudrik

Erik M. Mueller

Paul Muhle-Karbe

Shradha Mukherjee

Christophe Mulle

Jeff S. Mumm

Geoffrey G. Murphy

Peter R. Murphy

Tim H. Murphy

Timothy Hugh Murphy

Elisabeth A. Murray

Jennifer E. Murray

Micah M. Murray

Aditya Murthy

Erik S. Musiek

Isabel A. Muzzio

Nicholas Edward Myers

Sadegh Nabavi

Lionel Naccache

Janice R. Naegele

Satish S. Nair

Masato Nakafuku

Yasushi Nakagawa

Atsushi Nambu

Anirvan Nandy

Thomas Naselaris

Matthew Nassar

Samuel Nastase

Ian Nauhaus

Christopher Navara

Víctor M. Navarro
Klaus-Armin Nave

Martin Paul Nawrot

Yuji Naya

Maiken Nedergaard

Derek Evan Nee

Alexandra Bronwen Nelson

Dezso Nemeth

Torbjørn V. Ness

Adrian Nestor

Casey C. Nestor

Harald Neumann

Joshua P. Neunuebel

Nora Newcombe

Fiona Nora Newell

Ehren L. Newman

Eric A. Newman

Chi T. Ngo

Charles Nicholson

Saleem M. Nicola

Teresa Nicolson

Andreas Nieder

Jens Bo Nielsen

Kristina J. Nielsen

Matthias Niemeier

Hendrikje Nienborg

Till Nierhaus

Freek Nieuwhof

Vadim V. Nikulin

Yuval Nir

Hisao Nishijo

Akiko Nishiyama

Michael A. Nitsche

Douglas Arthur Nitz

Toemme Noesselt

Matthew Nolan

Anthony M. Norcia

Kenneth A. Norman

Sam Victor Norman-Haignere

Claus Normann

Georg Northoff

William Norton

Lucia Notterpek

Behrad Noudoost

Kirill V. Nourski

Manuela Nowotny

Daichi Nozaki

Silke Nuber

Michael D. Nunez

Michael P. Nusbaum

Zoltan Nusser

M. Kerry O'Banion

John O'Brien

Emily Oby

Redmond G. O'Connell

John J. O'Connor

Kate M. O'Connor-Giles

Michael J. O'Donovan

Wolgang H. Oertel

Thomas G. Oertner

Yulia Oganian

Damien O

'Halloran

Kenichi Ohki

Kathrin Ohla

Makoto Ohmoto

Masuo Ohno

Yukihiro Ohno

Yoshitaka Oka 
Ken-Ichi Okamoto

Hideyuki Okano

Michael Okun

Freyja Olafsdottir

Foster Olive

Dominik Oliver

Douglas L. Oliver

Cheryl A. Olman

Michelle Lynn Olsen

Shawn R. Olsen

Ingrid R. Olson

Leonie Oostwoud Wijdenes

Jean-Jacques Orban de Xivry

Lauren L. Orefice

Harry T. Orr

Amy Orsborn

Caitlin Orsini

Silvia Ortiz-Mantilla

Leslie C. Osborne

Sean Ostlund

Anne-Marie Michelle Oswald

James M. Otis

Swidbert Roger Ott

Ross Otto

Yvonne $\mathrm{Ou}$

Jackson C. Overath

Simon Alexander Overduin

Trevor Owens

Andrew J. Oxenham

Asli Ozyurek

Marius Pachitariu

Krishnan Padmanabhan

Pablo M. Paez

Kalipada Pahan

Sonia Paixao

Sarah L. Pallas

Valentina Pallottini

Lucy M. Palmer

Richard D. Palmiter

Daniela J. Palombo

Jorge J. Palop

Yannis Paloyelis

Amy Marie Palubinsky

Zhuo-Hua Pan

Theofanis I. Panagiotaropoulos

Zhiping Pang

Tina Pangrsic

Stefano Panzeri

Stella M. Papa

Suzanne Paradis

Michael A. Paradiso

Jack M. Parent

Vinay Parikh

Cesare Parise

Hyojin Park

Jeongho Park

Joonkoo Park

Kevin K. Park

Soojin Park

Krystal L. Parker

Shauna L. Parkes

Marie-Laure Parmentier

Josef Parvizi

Jeroen Pasterkamp

Alexandre Pastor-Bernier

Anitha Pasupathy

Eva Zita Patai

Ardem Patapoutian
Manisha Patel

Martin Paukert

Paul Pauli

Jeanne Tamar Paz

Rony Paz

Robert A. Pearce

Jonathan E. Peelle

Elior Peles

Giuseppe Pellizzer

Jukka Peltola

Jose L. Pena

Anthony W. Peng

Cyriel M. A. Pennartz

Peter Penzes

Mario Penzo

Monica A. Perez

Isabel Perez-Otaño

Brian D. Perkins

Simon Peron

Laurent Perrinet

Muriel Perron

Nora I. Perrone-Bizzozero

Abigail L. Person

Luiz Pessoa

Franco Pestilli

Laure Peter-Derex

Benjamin Peters

Jan Peters

Lien Peters

Rasmus Strange Petersen

Christopher I. Petkov

Leopoldo Petreanu

Lucy S. Petro

Timothy Petros

Jeffrey C. Petruska

Adrien Peyrache

Hans-Joachim Pflueger

Marios G. Philiastides

Polyxeni Philippidou

James Phillips

Kimberley A. Phillips

Mary Phillips

Vitoria Piai

Xianhua Piao

Elise Piazza

Jan Pielage

Alessandra Pierani

Jonathan T. Pierce-Shimomura

Richard Piet

Pietro Pietrini

Michele Pignatelli

Jonathan Pillow

Dimitris Pinotsis

Emmanuel Pinteaux

Payam Piray

Marc Pisansky

David Pitcher

Xaq Pitkow

Christopher Pittenger

Quentin J. Pittman

Diego A. Pizzagalli

Hilke Plassmann

David Pleasure

Samuel J. Pleasure

Burkhard Pleger

Kristen Pleil

Nikolaus Plesnila

Andrew John Robert Plested
Mikhail Pletnikov

Yannick Poitelon

Rafael Polania

Russell A. Poldrack

Alon Poleg-Polsky

Franck Polleux

Daniel B. Polley

Nicholas P. Poolos

Brian Popko

Maurizio Popoli

Tzvetan Popov

Gilles Pourtois

Petra Pouwels

Craig Motlow Powell

Jonathan Power

Albert Russell Powers

Lucas Pozzo-Miller

Peter Praamstra

Amynah A. A. Pradhan

Marco A. M. Prado

Murali Prakriya

Thomas Preat

Jochen H. M. Prehn

Steven A. Prescott

Clare Press

Cathy J. Price

Nicholas Seow Chiang Price

Nicholas J. Priebe

Emmanuel Procyk

Alex Proekt

Amy L. Proskovec

Mario Prsa

J. Andrew Pruszynski

Serge Przedborski

Edward N. Pugh

Jason R. Pugh

Sebastian Puschmann

Sathyanarayanan V. Puthanveettil

Teresa Puthussery

Daniela Puzzo

Sonja J. Pyott

Ling Qi

Shaozheng Qin

MengSheng Qiu

Christian Quaia

Anita M. Quintana

Gregory J. Quirk

Rodrigo Quian Quiroga

Jacob Raber

Gil D. Rabinovici

Angela Radulescu

Victor Frank Rafuse

Michael E. Ragozzino

David Raible

Sarah Raissi

Anjali M. Rajadhyaksha

Reza Rajimehr

Indira M. Raman

Siddharth Ramanan

Mani Ramaswami

Fernando M. Ramirez

Jan-Marino Ramirez

Amy J. Ramsey

Richard Ramsey

Charan Ranganath

Antonio Rangel

Hengyi Rao

Meenakshi Rao
Priyanka Rao-Ruiz

Matthew Rasband

Björn Rasch

Francois Rassendren

Supratim Ray

Lynn A. Raymond

Pamela A. Raymond

Khaleel A. Razak

Jennifer C. A. Read

Zachariah M. Reagh

Nelson Rebola

Gregg H. Recanzone

Leila Reddy

Geraint Rees

Wade G. Regehr

Tobias Reichenbach

Aylin Y. Reid

Kathryn J. Reissner

Maarten E. A. Reith

Jens Rekling

Joao Relvas

Luke R. Remage-Healey

Leonardo Restivo

Uwe Reuter

Antti Revonsuo

Alex D. Reyes

Ariane E. Rhone

Carlos Ribero

Anthony J. Ricci

Martin Riccomagno

Benjamin D. Richardson

Guy P. Richardson

Rick Richardson

Barry J. Richmond

Franziska Richter

Hermann Riecke

Florian Riese

Filippo Rijli

Johanna Maria Rimmele

Matthias Ringkamp

John Rinzel

Maureen Ritchey

Brendan Ritchie

Timothy Rittman

Harrison Ritz

David M. Robbe

Erik D. Roberson

Michael Thomas Roberts

Todd F. Roberts

Caroline E. Robertson

Janice Robertson

Jessica Robin

Alfred Jay Robison

Richard Robitaille

Jose Rodriguez-Alvarez

Pieter Roelf Roelfsema

Karin Roelofs

Matthew R. Roesch

Timothy Thomas Rogers

Tibor Rohacs

Tim Rohe

Marc Roig

Jamie D. Roitman

Martin Rolfs

Melissa M. Rolls

Gregg W. Roman

Lizabeth M. Romanski

Heinrich Römer 
Dorit Ron

Avtar Roopra

David H. Root

Marcello G. P. Rosa

Jeffrey B. Rosen

Merri J. Rosen

Stuart Rosen

Ari Rosenberg

Monica D. Rosenberg

Paul A. Rosenberg

Miriam Rosenberg-Lee

Kobi Rosenblum

Christian Rosenmund

Susanna Rosi

Sarah E. Ross

William N. Ross

Simone Rossi

Bruno Rossion

Stephanie Rossit

Adrian Rothenfluh

John Rothwell

Patrick Rothwell

Michael J. Rowan

Benjamin Andrew Rowland

John Rubenstein

Jonathan Rubin

Maria Eulalia Rubio

Christian Ruff

Douglas Andrew Ruff

Michael Rugg

Gavin Rumbaugh

Simon Rumpel

Rainer Rupprecht

Matthew F. S. Rushworth

Brian Russ

Andrew F. Russo

Scott J. Russo

Edward S. Ruthazer

Mark A. Rutherford

Ueli Rutishauser

Lukas Rüttiger

Manuela Ruzzoli

Timothy A. Ryan

William Rymer

David K. Ryugo

Yuri B. Saalmann

Laura Saba

Bernardo L. Sabatini

Silke Sachse

Todd Charlton Sacktor

Sepideh Sadaghiani

Martin J. Sadowski

Boris V. Safronov

Alvaro Sagasti

Armen Saghatelyan

Pankaj Sah

Amar Sahay

Mustafa Sahin

Minoru Saitoe

Amirsaman Sajad

Norio Sakai

Jon T. Sakata

Shuzo Sakata

John Sakon

Katsuyasu Sakurai

Carlo Sala

Alireza Salami

Armando Salinas
Jerome Sallet

Roy Salomon

Daniela Salvemini

Jason Samaha

Gregory Russell Samanez-Larkin

Daniela Sammler

Alapakkam P. Sampath

Benjamin Adam Samuels

Maria V. Sanchez-Vives

Myriam Christine Sander

Carmen Sandi

Jürgen Sandkühler

Joshua R. Sanes

Susan Sangha

Marco Santello

Viji Santhakumar

Clifford B. Saper

Przemyslaw Sapieha

H. Uri Saragovi

Marco Sardiello

Ilker Kudret Sariyer

Fabrice R. Sarlegna

Johannes Sarnthein

Martin Sarter

Magdalena Sastre

Tatsuo Sato

Ajay B. Satpute

Theodore Satterthwaite

Rita Gabriele Sattler

Frédéric Saudou

Benjamin Saunders

Nicola Savill

Iaroslav Alex Savtchouk

Nathaniel Sawtell

Rebecca Saxe

Zeynep M. Saygin

Christian P. Schaaf

Manuel Schabus

Andreas T. Schaefer

Anne Schaefer

Roland Schaette

Dorothy Schafer

Jeffrey Schall

Anna C. Schapiro

Constance Scharff

Helen E. Scharfman

Mathias Scharinger

Natalie Schaworonkow

Peter Scheiffele

Hansjörg Scherberger

Giampietro Schiavo

Ueli Schibler

Marc H. Schieber

Alejandro F. Schinder

Michael Schlossmacher

Michael Schmid

Tiffany Schmidt

Christoph Schmidt-Hieber

Ralf Schneggenburger

Keith A. Schneider

Peter Schneider

Eric Schnell

Jan Wilbert Hendrick Schnupp

Susanne Schoch

Timothy J. Schoenfeld

Benjamin Scholl

Marieke L. Schölvinck

David Schoppik
Stephanie Schorge

Laura A. Schrader

Christoph E. Schreiner

Timm Schubert

Nicolas W. Schuck

Oren Schuldiner

Heidrun Schultz

Aaron Schurger

Carol Schuurmans

Martin E. Schwab

Markus Schwaninger

Andrew B. Schwartz

Michal Schwartz

Michael Schwartze

Cornelius Schwarz

Jaclyn M. Schwarz

Dietrich Samuel Schwarzkopf

Nicolas Schweighofer

Felix E. Schweizer

Annalisa Scimemi

Rebecca P. Seal

Jeremy K. Seamans

Willam Sedley

Mohamed L. Seghier

Philippe Séguéla

Rachael Seidler

Elena Seiradake

Aaron R. Seitz

Kazuhiko Seki

Luc P. J. Selen

Matthew William Self

Frank Sengpiel

Stefano Sensi

John Serences

Martin I. Sereno

Andrea Serino

Nenad Sestan

Laurent Seugnet

Steven Shabel

Alexander J. Shackman

Reza Shadmehr

Mala M. Shah

Shihab A. Shamma

Robert Shapley

Reza Sharif-Naeini

Madeleine Sharp

Paul Sharp

Melissa J. Sharpe

Carla J. Shatz

Paul J. Shaw

Lavinia Sheets

Mark Sheffield

David L. Sheinberg

Signy Sheldon

Jie Shen

Amitai Shenhav

Jason Shepherd

Graham K. Sheridan

Larry Scott Sherman

S. Murray Sherman

Tomomi Shimogori

Jung Hoon Shin

Jung-Bum Shin

Minoru Shinohara

Priyattam J. Shiromani

Esther Shohami

Sarah Shomstein

Prerana Shrestha
Veronica I. Shubayev

Gordon L. Shulman

Claude William Shuttleworth

Francesca Siclari

Hartwig Roman Siebner

Steven A. Siegelbaum

Julie A. Siegenthaler

Amanda Sierra

Marion Silies

Roy Sillitoe

Edward Harry Silson

Michael Andrew Silver

Fraser J. Sim

Richard Simerly

Jonathan Z. Simon

Mikael Simons

Péter Simor

James W. Simpkins

Lawrence C. Sincich

Joshua H. Singer

Anton Sirota

Michael Artur Skeide

Frances Skinner

Erika Skoe

Efthimios M. C. Skoulakis

Heleen A. Slagter

Brianna Sleezer

Jamie Sleigh

Paul A. Slesinger

Dana M. Small

Jonathan Smallwood

Jeroen Smeets

Karen Janet Smillie

Douglas H. Smith

George M. Smith

Gordon Smith

Jeffrey C. Smith

Kyle Stephen Smith

Matthew A. Smith

Sheryl S. Smith

Stephen Mark Smith

Yoland Smith

Jason Scott Snyder

Abraham Z. Snyder

Adam Snyder

NaYoung So

Alexander I. Sobolevsky

Vikaas Singh Sohal

Samuel Solomon

Ken Solt

David C. Somers

Shinichi Someya

Marc A. Sommer

Wolfgang H. Sommer

Juan Song

Bo-mi Song

Barbara A. Sorg

Wayne S. Sossin

Danny Spampinato

Dennis R. Sparta

Karsten Specht

Alan C. Spector

Jeffrey L. Spees

Marc Spehr

Rebecca M. C. Spencer

Daniel J. Spergel

Jeffrey M. Spielberg

R. Nathan Spreng 
Rolf Sprengel

Andreas Sprenger

Krishnan Sriram

Deepak Prakash Srivastava

Robert W. Stackman

Mirre Stallen

Matthias Stangl

Bernhard P. Staresina

Philip A. Starr

Kathy Steece-Collier

Leonidas Stefanis

Wolfgang Stein

Marco Steinhauser

Nicholas A. Steinmetz

Mitchell Steinschneider

David Stellwagen

Deborah L. Stenkamp

Klaas Enno Stephan

Jeanne Ster

Philipp Sterzer

Charles F. Stevens

Bryan Stewart

Chelsea Stillman

Sean D. Stocker

Esther T. Stoeckli

Viola S. Stoermer

Arjen Stolk

Ruth L. Stornetta

Stefan Strack

Hans Straka

Andrew M. Strassman

David S. Strayer

Elizabeth Strickland

Ella Striem-Amit

Ben W. Strowbridge

Michael P. Stryker

Greg J. Stuart

Garret Stuber

Veit Stuphorn

Karuna Subramaniam

Thomas C. Südhof

Hoonkyo Suh

Jane Madden Sullivan

David Sulzer

Christopher Summerfield

Wei Sun

Mriganka Sur

Keiichiro Susuki

Michael A. Sutton

John Svaren

Alan F. Sved

Karel Svoboda

Nicole C. Swann

Geoffrey T. Swanson

Russell H. Swerdlow

Stephan P. Swinnen

János Szabadics

Julieta Sztarker

Denes Szucs

Karen Kathleen Szumlinski

Paul Taghert

Yuji K. Takahashi

Kaori Takehara-Nishiuchi

Tomonori Takeuchi

William T. Talman

Adolfo E. Talpalar

Arielle Tambini

Huiling Tan
Teruyuki Tanaka

Yamei Tang

Michael Tangermann

Heikki Tanila

Hiromu Tanimoto

Malú G. Tansey

Huizhong W. Tao

Victor Tarabykin

Michael J. Tarr

Jeffrey G. Tasker

Jessica Taubert

Janet L. Taylor

Jordan A. Taylor

Thomas E. Taylor-Clark

Tobias Teichert

Antonio L. Teixeira

Hugo Tejeda

Ludovic Telley

Vadim S. Ten

Sanne ten Oever

Xiangbin Teng

Andrea J. Tenner

James M. Tepper

Ei Terasawa

Melissa Terpstra

Alexey Terskikh

Giuseppina Tesco

Wolfram Tetzlaff

Preston P. Thakral

Jan Theeuwes

Alexander Thiele

Tod Thiele

Todd E. Thiele

Gopal Thinakaran

Scott M. Thompson

Peter R. Thorne

Mark Allen Thornton

David Thura

Gregor Thut

Fadel Tissir

Seema Tiwari-Woodruff

Philippe Tobler

Andrew J. Todd

Rebecca Todd

Slobodan M. Todorovic

George Tofaris

Shubha Tole

Kimberley F. Tolias

Seth M. Tomchik

Alexa Tompary

Glenn Matthew Toney

Frank Tong

Roger B. H. Tootell

Mary M. Torregrossa

Carole Torsney

Katalin Toth

Philip Tovote

Stephen Michael Town

Terrence Town

Dan Tracey

Robert Trampel

Susan P. Travers

Michael Tilghman Treadway

Alexandra Nicole Trelle

Andrew James Trevelyan

Elizabeth Tricomi

James S. Trimmer
Marco Tripodi

Nicholas F. Trojanowski

Sophie Tronel

Natalie C. Tronson

Vincent Tropepe

Ian A. Trounce

Laurence O. Trussell

Li-Huei Tsai

Nien-Pei Tsai

Peter Tsai

Albert Tsao

Richard Tsien

Makoto Tsuda

Susan Tsunoda

Kamen A. Tsvetanov

Stephen Tucker

Eugene Tunik

Nicholas B. Turk-Browne

Peter E. Turkeltaub

David L. Turner

Eric E. Turner

Glenn C. Turner

Ray W. Turner

Gina Turrigiano

John C. Tuthill

Douglas B. Tweed

Lorraine K. Tyler

Thanos Tzounopoulos

Yasuo Uchiyama

Lucina Q. Uddin

Péter P. Ujma

Markus Ullsperger

Henning Ulrich

Nash Unsworth

Janice H. Urban

Yuriy M. Usachev

Ted B. Usdin

W. Martin Usrey

Victor V. Uteshev

Jada Vaden

David E. Vaillancourt

Gregorio Valdez

Carlos Fernando Valenzuela

Taufik A. Valiante

Giorgio Vallortigara

Jeroen van Baar

Ruud Lucas Van den Brink

Martijn Pieter van den Heuvel

Michel Christian van den Oever

Wim Van Drongelen

Freek van Ede

Russell N. Van Gelder

Stephen David Van Hooser

Victor S. Van Laar

Dirk van Moorselaar

Vonne Van Polanen

Henriette van Praag

Carien M. van Reekum

David L. Van Vactor

Guido van Wingen

Christine Vande Velde

Louk J. M. J. Vanderschuren

Wim Vanduffel

Sampsa Vanhatalo

Tim Vanmierlo

Seralynne Denise Vann

Divya Varadarajan

Robert Vassar
Eliana Vassena

Deniz Vatansever

Christopher Walter Vaughan

Elena M. Vazey

Leandro F. Vendruscolo

Marco Venniro

Dineke S. Verbeek

Mieke Verfaellie

Valerie M. K. Verge

Matthijs Verhage

Lennart Verhagen

Eric Verschooten

Willem Verwey

Michael Vesia

Gisella Vetere

Jonathan D. Victor

Andrea Viczian

Imre Vida

Andres Vidal-Gadea

Susheel Vijayraghavan

Luis Villanueva

Roberto Vincis

Martin Vinck

Kasper Vinken

Kristina Visscher

Thomas Voets

Laura Anne Volpicelli-Daley

Katharina von Kriegstein

Mark Von Zastrow

Joel Lawrence Voss

Keith A. Vossel

Bradley Voytek

Serge Vulliemoz

Vladyslav V. Vyazovskiy

Matt Wachowiak

Scott Waddell

Alex Robert Wade

Anthony D. Wagner

John J. Wagner

Claes Wahlestedt

Claire-Dominique Walker

Kerry Marie May Walker

Matthew Charles Walker

Robin Walker

Judith R. Walters

Edgar T. Walters

Dirk B. Walther

Matthew J. Wanat

Dong V. Wang

Fan Wang

Jing Wang

Jun Wang

Kuan Wang

Xinnan Wang

Yu Tian Wang

Susan G. Wardle

Christopher Warren

Leonhard Waschke

Michael Waskom

Mitsuko Watabe-Uchida

Masahiko Watanabe

Trent Watkins

Andrew Watrous

Douglas J. Weber

Franz Weber

Elise Weerts

Richard J. Weinberg

Jeff L. Weiner 
David Weinshenker

Catherine J. C. Weisz

Nathan Weisz

Andrew E. Welchman

Cara L. Wellman

Tanya Wen

Theodore G. Wensel

Markus Werkle-Bergner

Jan R. Wessel

Martin W. Wessendorf

Daniel W. Wesson

Andrew Westbrook

Anne L. Wheeler

Patrick J. Whelan

Ian Q. Whishaw

Brian J. White

Fletcher A. White

Patricia M. White

Stephanie Ann White

Stuart F. White

Jonathan Whitlock

Sarah Whittle

Hynek Wichterle

Heinz Wiendl

Martin Wiener

Alex I. Wiesman

Linda Wilbrecht

Jill Wildonger

Roel M. Willems

John T. Williams

Michael T. Williams

Stephen R. Williams

Ziv M. Williams

Dianna Willis

Charles J. Wilson

Donald A. Wilson

Matt Wilson

Robert C. Wilson

Stephen M. Wilson

Tony W. Wilson

Maria Wimber

G. Elliott Wimmer

Mathieu E. Wimmer
Bettina Winckler

Danny G. Winder

Juergen Winkler

Raphaelle Winsky-Sommerer

Marta Barbara Wisniewska

Gábor Wittmann

Magdalena Wojtczak

Martijn E. Wokke

Marina E. Wolf

Oliver Tobias Wolf

Uta Wolfe

Mathieu Wolff

Herman Wolosker

Benjamin Wolozin

Thilo Womelsdorf

Aaron Wong

Jennifer C. Wong

Philip C. Wong

Tak Pan Wong

Guilherme Wood

Marcelo A. Wood

Nicholas Isaiah Woods

John J. Woodward

Alex Woolgar

Sarah Cushing Woolley

Sarah M. N. Woolley

Malte Wöstmann

Chengbiao Wu

Ling-Gang Wu

Long-Jun $\mathrm{Wu}$

Mingzheng Wu

Moritz F. Wurm

Andreas Wutz

Frances Xia

Ruili Xie

Wendy Xin

Yan Xing

Huaxi Xu

Junqian Xu

Yaoda Xu

Matthew Alexander Xu-Friedman

Essa Yacoub

Jerrel L. Yakel
Emre Yaksi

Junji Yamauchi

Riqiang Yan

Tianming Yang

Yongjie Yang

Yunlei Yang

Wei-Dong Yao

Tal Yarkoni

Avraham Yaron

Ryohei Yasuda

Jeffrey M. Yau

Gary Yellen

B. T. Thomas Yeo

Christopher Yeo

Jerry C. P. Yin

Atsushi Yokoi

Keisuke Yonehara

V. Wee Yong

Sung Ok Yoon

Yutaka Yoshida

Takanobu Yoshii

Kaylene M. Young

Larry Young

Samuel Young, Jr.

Galit Yovel

Eric A. Yttri

Fengwei Yu

Guoqiang Yu

Rongjun Yu

Quan Yuan

Zhenyu Yue

Shlomit Yuval-Greenberg

Venetia Zachariou

Edward Zagha

Kareem A. Zaghloul

Daniel S. Zahm

Stanislav S. Zakharenko

Harold H. Zakon

Abraham Zangen

Theodore Zanto

Baltazar Antonio Zavala

Dasa Zeithamova

Scott O. Zeitlin
Christina Zelano

Moriel Zelikowsky

Lori Zeltser

David Zenisek

Chun-Li Zhang

Han Zhang

Jiaxiang Zhang

Jinsheng Zhang

John H. Zhang

Jun-Ming Zhang

Li I. Zhang

Mingjie Zhang

Yanfeng Zhang

Yun Zhang

Zheng Gang Zhang

Bin Zhao

Li Zhaoping

Mei Zhen

Sika Zheng

Feng-Quan Zhou

Fu-Ming Zhou

Jingyang Zhou

Qiang Zhou

Yi Zhou

Haining Zhu

Yongling Zhu

Yunlu Zhu

Xiaoxi Zhuang

Inge Zijdewind

Kai Zinn

Elana Zion-Golumbic

Karen Zito

Benedikt Zoefel

Huda Y. Zoghbi

Wen-Quan Zou

Iva Zovkic

Jon-Kar Zubieta

Brad Zuchero

Michaël B. Zugaro

R. Suzanne Zukin

Damian G. Zuloaga

Jian Zuo

Daniel Zytnicki 